$\begin{array}{cccc}\begin{array}{c}\text { Laboratory } \\ \text { number }\end{array} & \begin{array}{c}\text { Published } \\ \text { reference }\end{array} & \begin{array}{c}\text { Original date or } \\ \text { other value }\end{array} & \begin{array}{c}\text { Corrected date } \\ \text { or other value }\end{array} \\ -85 & 125: 85 & 430 \pm 90 & \\ -86 & 125: 85 & 370 \pm 80 & \\ -92 \mathrm{~A} & 125: 82 & 1890 \pm 90 & \\ -92 \mathrm{~B} & 125: 82 & 1850 \pm 85 & \\ -93 & 167: 218 & 200 \pm 200 & \\ -94 \mathrm{~A} & 167: 214 & 2050 \pm 50 & \\ -94 \mathrm{~B} & 167: 214 & 2440 \pm 55 & \\ -95 & 167: 213 & 5825 \pm 75 & \\ -96 & 167: 214 & 3230 \pm 60 & \\ -97 & 167: 215 & 3475 \pm 60 & \\ -98 & 167: 215 & 3075 \pm 60 & \\ -106^{4} & 125: 79 & 11,800 \pm 280 & \\ -109 & 167: 215 & 7800 \pm 100 & \\ -113 & 125: 80 & 2100 \pm 80 & \\ -114 & 125: 80 & 2970 \pm 90 & \\ -117 & 167: 217 & 3100 \pm 60 & \\ -122 & 125: 81 & 4650 \pm 90 & \\ -130 & 167: 218 & 13,170 \pm 175 & \end{array}$

A.D./

B.C. date

A.D. 1520

A.D. 1580

A.D. 60

A.D. 100

A.D. 1750

100 B.C.

490 B.C.

3875 B.C

1280 B.C.

1525 B.C.

1125 B.C.

9850 B.C.

5850 B.C

150 B.C.

1020 B.C.

1150 B.C.

2700 B.C.

11,220 B.C.

${ }^{1}$ Reference standard: Wood grown near Rome between 1949 and 1953.

${ }^{2}$ Cross-check sample (R-1) distributed by Rome Lab. to many laboratories: T-9; St103/A/B； BM-15; Q-112; U-68

${ }^{3}$ R-64, cross-check sample distributed by H. Tauber, Copenhagen Lab., (K-101) to many labs.: K-101 bis; K-102 bis; Gro-454; St-18; U-20; U-75; H-105; BM-19; W-82, W-84.

${ }^{4}$ Sample given us by Hl. de Vries at Groningen (Nov. 1957); it is the sample pub. as Gro-933 and Gro-948.

${ }^{5}$-Pub. in Quaternaria, v. 5, p. 94.

\title{
University of Saskatchewan, Department of Chemistry
}

\begin{tabular}{|c|c|c|c|c|c|}
\hline $\begin{array}{l}\text { Laboratory } \\
\text { number }\end{array}$ & $\begin{array}{l}\text { Published } \\
\text { reference }\end{array}$ & $\begin{array}{l}\text { Original date or } \\
\text { other value }\end{array}$ & $\begin{array}{l}\text { Corrected date } \\
\text { or other value }\end{array}$ & $\partial \mathrm{C}^{14}, \% 0$ & $\begin{array}{c}\text { A.D./ } \\
\text { B.C. date }\end{array}$ \\
\hline S-1 & 180 & $1910 \pm 130^{\mathrm{s}}$ & & $211 \pm 17$ & A.D. 40 \\
\hline-2 & 180 & $3400 \pm 200^{s}$ & $2780 \pm 200 \mathrm{c}$ & $292 \pm 16$ & 830 B.C. \\
\hline-3 & 180 & $2430 \pm 160^{\mathrm{s}}$ & & $260 \pm 16$ & 480 B.C. \\
\hline-4 & 180 & $2415 \pm 160^{\mathrm{s}}$ & & $268 \pm 16$ & 465 B.C. \\
\hline-7 & 180 & $>25,000^{\mathrm{s}}$ & & $966 \pm 16$ & \\
\hline-12 & 180 & $3670 \pm 270^{\mathrm{s}}$ & & $367 \pm 21$ & 1720 B.C. \\
\hline-13 & 180 & $5600 \pm 300^{\mathrm{s}}$ & & $502 \pm 21$ & 3650 B.C. \\
\hline-16 & $89: 73$ & $6250 \pm 250$ & $6300 \pm 250 \mathrm{a}$ & $544 \pm 11$ & 4350 B.C. \\
\hline-17 & 180 & d & see $\mathrm{S}-17 \mathrm{bis}$ & & \\
\hline-17 bis & $89: 77$ & $2350 \pm 60$ & & $252 \pm 6$ & 400 B.C. \\
\hline-18 & $89: 77$ & $2450 \pm 160$ & & $264 \pm 11$ & 500 B.C. \\
\hline-19 & $89: 77$ & $1580 \pm 140$ & & $179 \pm 12$ & A.D. 370 \\
\hline .20 & $89: 77$ & $660 \pm 130$ & & $80 \pm 10$ & A.D. 1290 \\
\hline-22 & 180 & $1580 \pm 159^{\mathrm{s}}$ & & $179 \pm 24$ & A.D. 370 \\
\hline-25 & 180 & $4400 \pm 216^{s}$ & $\mathrm{~d}$ & $438 \pm 23$ & \\
\hline$-25^{1}$ & $89: 74$ & $4600 \pm 210$ & $4650 \pm 210 \mathrm{a}$ & $439 \pm 15$ & 2700 B.C. \\
\hline .26 & 180 & $>23,000^{\mathrm{s}}$ & & $1015 \pm 28$ & \\
\hline .28 & 180 & $2400 \pm 173^{\mathrm{s}}$ & & $258 \pm 24$ & 450 B.C. \\
\hline-29 & $89: 74$ & $11,400 \pm 450$ & & $760 \pm 11$ & 9450 B.C. \\
\hline-30 & $89: 74$ & $12,000 \pm 500$ & & $777 \pm 11$ & 10,050 B.C. \\
\hline-31 & 180 & $12,660 \pm 440^{\mathrm{s}}$ & & $793 \pm 21$ & 10,710 B.C. \\
\hline-32 & $90: 71$ & $7800 \pm 300$ & $7850 \pm 300 \mathrm{a}$ & $622 \pm 11$ & 5900 B.C. \\
\hline-33 & $90: 71$ & $2980 \pm 175$ & $3030 \pm 175$ a & $313 \pm 11$ & 1080 B.C. \\
\hline-34 & $90: 71$ & $8180 \pm 320$ & $8230 \pm 320 \mathrm{a}$ & $670 \pm 12$ & 6280 B.C. \\
\hline-40 & $89: 76$ & $>33,000$ & & VJJD $\pm v C$ & \\
\hline-4.1 & $89: 74$ & $10,000 \pm 300$ & & $710 \pm 13$ & 8050 B.C. \\
\hline-42 & $89: 76$ & $>30,000$ & & $992 \pm 13$ & \\
\hline-43 & $89: 79$ & $400 \pm 120$ & $450 \pm 120 \mathrm{a}$ & $43 \pm 11$ & A.D. 1600 \\
\hline
\end{tabular}




\begin{tabular}{|c|c|c|c|c|c|}
\hline $\begin{array}{l}\text { Laboratory } \\
\text { number }\end{array}$ & $\begin{array}{l}\text { Published } \\
\text { reference }\end{array}$ & $\begin{array}{l}\text { Original date or } \\
\text { other value }\end{array}$ & $\begin{array}{l}\text { Corrected date } \\
\text { or other value }\end{array}$ & $\partial \mathrm{C}^{14}, \% 0$ & $\begin{array}{l}\text { A.D./ } \\
\text { B.C. date }\end{array}$ \\
\hline-44 & $89: 80$ & $5200 \pm 130$ & & $477 \pm 12$ & 3250 B.C. \\
\hline-45 & $89: 80$ & $1500 \pm 100$ & & $169 \pm 18$ & A.D. 450 \\
\hline$-46^{2}$ & $89: 74$ & $>34,000$ & & $1004 \pm 13$ & \\
\hline-47 & $89: 78$ & $8150 \pm 300$ & & $634 \pm 7$ & 6200 B.C. \\
\hline-48 & $89: 79$ & $4550 \pm 200$ & & $433 \pm 10$ & 2600 B.C. \\
\hline$-49 a$ & $90: 75$ & $2230 \pm 100$ & & $243 \pm 12$ & 280 B.C. \\
\hline$-49 \mathrm{~b}$ & $90: 75$ & $3710 \pm 70$ & & $370 \pm 7$ & 1760 B.C. \\
\hline-50 & $90: 75$ & $4620 \pm 150$ & & $437 \pm 10$ & 2670 B.C. \\
\hline-52 & $90: 75$ & $4620 \pm 80$ & & $436 \pm 6$ & 2670 B.C. \\
\hline-53 & $90: 76$ & $4650 \pm 150$ & & $439 \pm 10$ & 2700 B.C. \\
\hline-54 & $90: 76$ & $5000 \pm 125$ & & $464 \pm 7$ & 3050 B.C. \\
\hline-55 & $90: 76$ & $>30,000$ & & $994 \pm 7$ & \\
\hline-60 & $89: 79$ & $2850 \pm 60$ & & $300 \pm 7$ & 900 B.C. \\
\hline-61 & $89: 78$ & $7350 \pm 150$ & & $596 \pm 10$ & 5400 B.C. \\
\hline-62 & $89: 78$ & $2270 \pm 100$ & & $228 \pm 10$ & 320 B.C. \\
\hline$-63 a$ & $90: 75$ & $3370 \pm 115$ & & $353 \pm 14$ & 1420 B.C. \\
\hline$-63 b$ & $90: 75$ & $4520 \pm 170$ & & $428 \pm 13$ & 2570 B.C. \\
\hline-64 & $89: 75$ & $3500 \pm 70 \mathrm{~d}$ & & $354 \pm 7$ & \\
\hline .64 & $89: 75$ & $2800 \pm 90 d$ & & $292 \pm 15$ & \\
\hline-65 & $89: 75$ & $>32,000$ & & $1003 \pm 10$ & \\
\hline-66 & $89: 80$ & $920 \pm 60$ & & $111 \pm 19$ & A.D. 1030 \\
\hline-67 & $89: 75$ & $200 \pm 120$ & & $25 \pm 20$ & A.D. 1750 \\
\hline-68 & $89: 75$ & $11,000 \pm 250$ & & $751 \pm 16$ & 9050 B.C. \\
\hline-73 & $89: 76$ & $1800 \pm 100$ & & $191 \pm 14$ & A.D. 150 \\
\hline-74 & $89: 76$ & $2900 \pm 100$ & & $301 \pm 10$ & 950 B.C. \\
\hline-87 & $89: 79$ & $1100 \pm 80$ & & $129 \pm 11$ & 850 B.C. \\
\hline-89 & $89: 79$ & $1020 \pm 80$ & & $121 \pm 12$ & 930 B.C. \\
\hline-90 & $90: 76$ & $1650 \pm 60$ & & $188 \pm 11$ & A.D. 300 \\
\hline .91 & $90: 76$ & $1840 \pm 70$ & & $206 \pm 10$ & A.D. 110 \\
\hline$-92^{3}$ & $89: 76$ & $>31,000$ & & $1005 \pm 15$ & \\
\hline .93 & $89: 77$ & $1780 \pm 60$ & & $199 \pm 9$ & A.D. 170 \\
\hline-94 & $90: 71$ & $3200 \pm 70$ & & $328 \pm 10$ & 1250 B.C. \\
\hline-96 & $90: 72$ & $27,750 \pm 1200$ & & $969 \pm 9$ & \\
\hline-97 & $90: 72$ & $10,150 \pm 200$ & & $718 \pm 8$ & 8200 B.C. \\
\hline$-99^{4}$ & $90: 73$ & $7300 \pm 120$ & & $599 \pm 9$ & 5350 B.C. \\
\hline-100 & $90: 74$ & $9570 \pm 150$ & & $697 \pm 8$ & 7620 B.C. \\
\hline-102 & $90: 76$ & $1650 \pm 70$ & & $184 \pm 8$ & A.D. 300 \\
\hline-103 & $90: 76$ & $3750 \pm 80$ & & $359 \pm 9$ & 1800 B.C. \\
\hline .104 & $90: 76$ & $400 \pm 60$ & & $49 \pm 8$ & A.D. 1550 \\
\hline-105 & $90: 76$ & $380 \pm 70$ & & $46 \pm 8$ & A.D. 1570 \\
\hline-106 & $90: 74$ & $8150 \pm 100$ & & $636 \pm 11$ & 6200 B.C. \\
\hline-107 & $90: 74$ & $7350 \pm 100$ & & $601 \pm 13$ & 5400 B.C. \\
\hline-108 & $90: 77$ & $835 \pm 70$ & & $99 \pm 6$ & A.D. 1115 \\
\hline .109 & $90: 77$ & $760 \pm 60$ & & $89 \pm 13$ & A.D. 1190 \\
\hline .110 & $90: 72$ & $10,300 \pm 140$ & & $722 \pm 7$ & 8350 B.C. \\
\hline-111 & $90: 72$ & $>30,000$ & & $995 \pm 13$ & \\
\hline-112 & $90: 77$ & $2360 \pm 60$ & & $251 \pm 3$ & 410 B.C. \\
\hline .113 & $90: 77$ & $9000 \pm 150$ & & $674 \pm 5$ & 7050 B.C. \\
\hline$-115 \mathrm{~A}$ & $90: 74$ & $5550 \pm 70$ & & $498 \pm 6$ & 3600 B.C. \\
\hline-116 & $90: 74$ & $8600 \pm 100$ & & $656 \pm 7$ & 6650 B.C. \\
\hline-117 & $90: 72$ & $>32,000$ & & $994 \pm 8$ & \\
\hline-119 & $90: 77$ & $2480 \pm 60$ & & $266 \pm 4$ & 530 B.C. \\
\hline-120 & $90: 78$ & $780 \pm 50$ & & $91 \pm 6$ & A.D. 1170 \\
\hline-121 & $90: 78$ & $260 \pm 50$ & & $17 \pm 6$ & A.D. 1690 \\
\hline-122 & $90: 73$ & $5050 \pm 80$ & & $466 \pm 6$ & 3100 B.C. \\
\hline-123 & $90: 73$ & $10,900 \pm 700$ & $10,900 \pm 170 \mathrm{c}$ & $743 \pm 7$ & 8950 B.C. \\
\hline-127 & $90: 73$ & $>34,000$ & & $996 \pm 7$ & \\
\hline-128 & $90: 73$ & $10,800 \pm 300$ & & $738 \pm 7$ & 8850 B.C. \\
\hline .129 & $90: 75$ & $9570 \pm 130$ & & $694 \pm 7$ & 7620 B.C. \\
\hline-132 & $169: 229$ & $550 \pm 80$ & & $66 \pm 5$ & A.D. 1400 \\
\hline .133 & $169: 229$ & $680 \pm 100$ & & $81 \pm 14$ & A.D. 1270 \\
\hline-134 & $169: 229$ & $1250 \pm 100$ & & $145 \pm 14$ & A.D. 700 \\
\hline
\end{tabular}




\begin{tabular}{|c|c|c|c|c|c|}
\hline $\begin{array}{c}\text { Laboratory } \\
\text { number }\end{array}$ & $\begin{array}{l}\text { Published } \\
\text { reference }\end{array}$ & $\begin{array}{l}\text { Original date or } \\
\quad \text { other value }\end{array}$ & $\begin{array}{l}\text { Corrected date } \\
\text { or other value }\end{array}$ & $\partial \mathrm{C}^{14}, \% 0$ & $\begin{array}{l}\text { A.D./ } \\
\text { B.C. date }\end{array}$ \\
\hline-135 & $169: 229$ & $1860 \pm 70$ & & $210 \pm 12$ & A.D. 90 \\
\hline-137 & $169: 229$ & $2050 \pm 100$ & & $225 \pm 11$ & 100 B.C. \\
\hline-138 & $90: 75$ & $2500 \pm 170$ & & $269 \pm 16$ & 550 B.C. \\
\hline-140 & $90: 74$ & $10,600 \pm 300$ & & $734 \pm 11$ & 8650 B.C. \\
\hline .146 & $169: 230$ & $>32,000$ & & $1011 \pm 9$ & \\
\hline-147 & $169: 230$ & $>33,000$ & & $1003 \pm 9$ & \\
\hline-148 & $169: 230$ & $245 \pm 60$ & & $30 \pm 8$ & A.D. 1705 \\
\hline-149 & $90: 78$ & $1220 \pm 80$ & & $140 \pm 13$ & A.D. 730 \\
\hline-150 & $90: 78$ & $1165 \pm 80$ & & $135 \pm 13$ & A.D. 785 \\
\hline-151 & $169: 230$ & $12,100 \pm 160$ & & $777 \pm 8$ & 10,150 B.C. \\
\hline-153 & $169: 234$ & $600 \pm 45$ & & $72 \pm 7$ & A.D. 1350 \\
\hline-154 & $169: 234$ & $900 \pm 50$ & & $108 \pm 8$ & A.D. 1050 \\
\hline-158 & $169: 234$ & $2125 \pm 65$ & & $232 \pm 7$ & 175 B.C. \\
\hline-161 & $169: 232$ & $6100 \pm 80$ & & $552 \pm 8$ & 4150 B.C. \\
\hline-162 & $169: 232$ & $7300 \pm 80$ & & $600 \pm 7$ & 5350 B.C. \\
\hline-164 & $169: 230$ & $1800 \pm 80$ & & $202 \pm 12$ & A.D. 150 \\
\hline-165 & $169: 230$ & $10,275 \pm 225$ & & $721 \pm 13$ & 8325 B.C. \\
\hline-172 & $169: 232$ & $12,000 \pm 200$ & & $772 \pm 10$ & 10,050 B.C. \\
\hline-173 & $169: 230$ & $13,000 \pm 200$ & & $800 \pm 9$ & 11,050 B.C. \\
\hline-174 & $169: 230$ & $10,250 \pm 150$ & & $716 \pm 9$ & 8300 B.C. \\
\hline-175 & $169: 231$ & $9250 \pm 150$ & & $684 \pm 6$ & 7300 B.C. \\
\hline-176 & $169: 231$ & $20,000 \pm 850$ & & $917 \pm 12$ & 18,050 B.C. \\
\hline-178 & $169: 231$ & $770 \pm 50$ & & $91 \pm 7$ & A.D. 1180 \\
\hline-179 & $169: 231$ & $470 \pm 50$ & & $55 \pm 7$ & A.D. 1480 \\
\hline .182 & $169: 231$ & $9600 \pm 120$ & & $695 \pm 7$ & 7650 B.C. \\
\hline-183 & $169: 234$ & $1290 \pm 75$ & & $148 \pm 12$ & A.D. 660 \\
\hline-184 & $169: 233$ & $2175 \pm 70$ & & $239 \pm 8$ & 225 B.C. \\
\hline-185 & $169: 233$ & $6850 \pm 100$ & & $573 \pm 7$ & 4900 B.C. \\
\hline .186 & $169: 233$ & $10,600 \pm 130$ & & $732 \pm 6$ & 8650 B.C. \\
\hline-187 & $169: 234$ & $500 \pm 60$ & & $61 \pm 7$ & A.D. 1450 \\
\hline-188 & $169: 232$ & $10,270 \pm 150$ & & $722 \pm 10$ & 8320 B.C. \\
\hline-189 & $169: 232$ & $10,630 \pm 150$ & & $736 \pm 10$ & 8680 B.C. \\
\hline-190 & $169: 232$ & $11,650 \pm 150$ & & $764 \pm 8$ & 9700 B.C. \\
\hline-191 & $169: 233$ & $6020 \pm 90$ & & $527 \pm 10$ & 4070 B.C. \\
\hline-198 & $169: 231$ & $12,140 \pm 240$ & & $779 \pm 13$ & 10,190 B.C. \\
\hline-208 & $169: 233$ & $410 \pm 150$ & & $53 \pm 16$ & A.D. 1540 \\
\hline-227 & $169: 231$ & $10,800 \pm 160$ & & $739 \pm 8$ & 8850 B.C. \\
\hline-301 & $90: 79$ & $\Delta-26 \pm 8$ & & & \\
\hline-302 & $90: 79$ & $\bar{\Delta}-28 \pm 8$ & & & \\
\hline-303 & $90: 79$ & $\Delta-4 \pm 8$ & & & \\
\hline-304 & $90: 79$ & $\Delta \quad-9 \pm 10$ & & & \\
\hline-305 & $90: 79$ & $\Delta \quad 73 \pm 6$ & & & \\
\hline-306 & $90: 79$ & $\vec{\Delta} \quad 107 \pm 7$ & & & \\
\hline-308 & $90: 79$ & $\Delta \quad 290 \pm 10$ & & & \\
\hline-309 & $90: 79$ & $\Delta-12 \pm 8$ & & & \\
\hline-310 & $90: 79$ & $\Delta \quad 184 \pm 11$ & & & \\
\hline-311 & $90: 79$ & $\Delta \quad 5 \pm 10$ & & & \\
\hline-313 & $90: 79$ & $\Delta \quad 176 \pm 10$ & & & \\
\hline-314 & $90: 79$ & $\Delta \quad 285 \pm 10$ & & & \\
\hline-315 & $90: 79$ & $\bar{\Delta} \quad 244 \pm 11$ & & & \\
\hline-316 & $90: 79$ & $\Delta \quad 320 \pm 10$ & & & \\
\hline-317 & $90: 79$ & $\Delta \quad 232 \pm 10$ & & & \\
\hline-318 & $90: 79$ & $\Delta \quad 238 \pm 10$ & & & \\
\hline-319 & $169: 235$ & $\Delta \quad 408 \pm 9$ & & & \\
\hline-320 & $169: 235$ & $\Delta \quad 452 \pm 10$ & & & \\
\hline-321 & $169: 235$ & $\Delta \quad 955 \pm 17$ & & & \\
\hline-322 & $169: 235$ & $\Delta \quad 913 \pm 10$ & & & \\
\hline $\begin{array}{l}{ }^{1} \text { GSC-20 } \\
{ }^{2} \mathrm{H}-440,2 \\
{ }^{3} \mathrm{Ma}-15, \\
{ }^{4} \mathrm{GSC}-2,\end{array}$ & $\begin{array}{l}275 \pm 100 . \\
00 ; \text { Gro- } 25 \\
600 \pm 900 . \\
00 \pm 150 .\end{array}$ & $00,44,200 \pm 1500$ & & & \\
\hline
\end{tabular}

